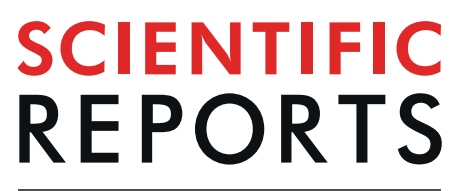

natureresearch

Check for updates

\title{
Non-pharmacological treatment changes brain activity in patients with dementia
}

\begin{abstract}
Yoshihito Shigihara $\mathbb{1}^{1,2}{ }^{\square}$, Hideyuki Hoshi ${ }^{1}$, Keita Shinada ${ }^{3}$, Toyoji Okada ${ }^{4}$ \& Hajime Kamada ${ }^{2}$
Non-pharmacological treatment (NPT) improves cognitive functions and behavioural disturbances in patients with dementia, but the underlying neural mechanisms are unclear. In this observational study, 21 patients with dementia received NPTs for several months. Patients were scanned using magnetoencephalography twice during the NPT period to evaluate NPT effects on resting-state brain activity. Additionally, cognitive functions and behavioural disturbances were measured using the MiniMental State Examination (MMSE-J) and a short version of the Dementia Behaviour Disturbance Scale (DBD-13) at the beginning and the end of the NPT period. In contrast to the average DBD-13 score, the average MMSE-J score improved after the NPT period. Magnetoencephalography data revealed a reduced alpha activity in the right temporal lobe and fusiform gyrus, as well as an increased lowgamma activity in the right angular gyrus. DBD-13 score changes were correlated with beta activity in the sensorimotor area. These findings corroborate previous studies confirming NPT effects on brain activity in healthy participants and people at risk of dementia. Our results provide additional evidence that brains of patients with dementia have the capacity for plasticity, which may be responsible for the observed NPT effects. In dementia, NPT might lead to improvements in the quality of life.
\end{abstract}

Dementia is a chronic and progressive syndrome caused by brain diseases ${ }^{1}$. It is characterised by deterioration in cognitive functions, behaviour, and psychological symptoms. To date, there are only a few pharmacological treatment options available to influence the course of dementia ${ }^{2-4}$. At the time a patient is diagnosed with dementia, the brain damage is considered too severe to be reversible to a healthy state ${ }^{5}$. Thus, early diagnosis and interventions are essential to treat people at risk of dementia, such as those with "cognitive impairment, no dementia" (CIND) or "mild cognitive impairment" $(\mathrm{MCI})^{6-9}$. Recent studies showed that MCI can be reversed to normal cognitive functions by non-pharmacological treatments (NPTs $)^{10-15}$. Neuroimaging studies revealed that NPTs enhance brain plasticity even in older adults ${ }^{16,17}$ supporting the importance of early diagnosis and interventions in dementia.

The positive effects of NPTs are not limited to people at risk such as those with CIND or MCI. Clinicians and therapists frequently observe that NPTs improve cognitive functions and/or behaviours in patients with dementia $^{18-23}$. However, the neural mechanisms underlying the positive influences of NPTs remain unclear.

Geriatric health service facilities are transitional facilities between hospital and home or nursing home where registered physical, occupational, and speech therapists provide NPT and nursing care to reduce the patients' hospital stay. Patients with dementia are often transferred there when their physical conditions are usually not too severe to be treated in hospitals but their cognitive symptoms prevent them from staying at their home. The primary goal of the facility is to improve the patients' behavioural functions, to enable them to live again at home ${ }^{21}$. Although cognitive improvements are the secondary target, patients in these facilities often show progress in cognitive functions, as well as in behavioural and psychological symptoms of dementia (BPSD) ${ }^{20}$. Based on these experiences, clinicians and therapists are often under the impression that patients with dementia still exhibit sufficient brain plasticity to respond to NPTs; however, evidence for this is sparse in literature. In this study, 21 patients with dementia were recruited at our geriatric health service facility. They received NPTs for several months and their resting-state brain activities were measured using magnetoencephalography (MEG) two times during the total NPT period. The brain activity recordings were compared to better understand how NPT changes the resting-state brain activities in patients with dementia.

${ }^{1}$ Precision Medicine Centre, Hokuto Hospital, Obihiro City, Japan. ${ }^{2}$ Department of Neurosurgery, Hokuto Hospital, Obihiro City, Japan. ${ }^{3}$ Geriatric Health Services Facility Kakehashi, Hokuto Hospital Group, Obihiro City, Japan. ${ }^{4}$ Department of Clinical Laboratory, Hokuto Hospital, Obihiro City, Japan. ${ }^{\bowtie}$ e-mail: y-shigihara@ @ hokuto7.or.jp 


\begin{tabular}{|c|c|c|c|c|c|c|c|c|c|c|c|c|c|}
\hline \multirow[b]{2}{*}{ ID } & \multirow[b]{2}{*}{ Age } & \multirow[b]{2}{*}{ Sex } & \multirow{2}{*}{\begin{tabular}{|l} 
Reported \\
Diagnose
\end{tabular}} & \multirow{2}{*}{\begin{tabular}{|l|}
$\begin{array}{l}\text { Interval of the } \\
\text { scans }\end{array}$ \\
(days) \\
\end{tabular}} & \multicolumn{3}{|c|}{ Initial score } & \multicolumn{3}{|c|}{ Final score } & \multicolumn{3}{|l|}{ Change } \\
\hline & & & & & Severity & MMSE & DBD-13 & Severity & MMSE & DBD-13 & Severity & MMSE & DBD-13 \\
\hline Average & 85.1 & & & 75.7 & 4.2 & 12.4 & 10.6 & 3.9 & 14.4 & 9.9 & -0.2 & 2.0 & -0.7 \\
\hline SD & 5.4 & & & 31.7 & 1.6 & 6.6 & \begin{tabular}{|l|l|}
10.8 \\
\end{tabular} & 1.6 & 7.8 & 11.1 & 0.5 & 3.2 & 2.7 \\
\hline Max & 94.0 & & & 133 & 6.0 & 22.0 & 43.0 & 6.0 & 29.0 & 40.0 & 0.0 & 8.0 & 7.0 \\
\hline Min & 73.0 & & & 28 & 2.0 & 0.0 & \begin{tabular}{|l|} 
\\
\end{tabular} & 2.0 & 0.0 & $\begin{array}{l}0.0 \\
\end{array}$ & -2.0 & -3.0 & -4.0 \\
\hline Median & 84.5 & & & 75 & 4.0 & 12.5 & 6.5 & 4.0 & 12.5 & 5.5 & 0.0 & 2.0 & -1.0 \\
\hline 1 & 73 & F & VD & 73 & 3 & 18 & 0 & 2 & 20 & 0 & -1 & 2 & 0 \\
\hline 2 & 79 & F & VD & 77 & 6 & 3 & 4 & 6 & 5 & 3 & 0 & 2 & -1 \\
\hline 3 & 79 & $\mathrm{M}$ & VD & 52 & 4 & 8 & 0 & 4 & 13 & 2 & 0 & 5 & 2 \\
\hline 4 & 80 & $\mathrm{~F}$ & VD & 95 & 2 & 21 & 2 & 2 & 21 & 1 & 0 & 0 & -1 \\
\hline 5 & 81 & F & Not specified & 91 & 6 & 0 & 43 & 6 & 0 & 40 & 0 & 0 & -3 \\
\hline 6 & 83 & $\mathrm{~F}$ & $\mathrm{AD}$ & 53 & 4 & 13 & 6 & 4 & 12 & 6 & 0 & -1 & 0 \\
\hline 7 & 84 & M & $\mathrm{AD}$ & 133 & 6 & 12 & 4 & 5 & 11 & 3 & -1 & -1 & -1 \\
\hline 8 & 84 & F & $\mathrm{AD}$ & 133 & 5 & 11 & 20 & 3 & 18 & 19 & -2 & 7 & -1 \\
\hline 9 & 84 & M & $\mathrm{AD}$ & 95 & 2 & 21 & 4 & 2 & 24 & 1 & 0 & 3 & -3 \\
\hline 10 & 85 & M & $\mathrm{AD}$ & 31 & 5 & 7 & 10 & 5 & 6 & 11 & 0 & -1 & 1 \\
\hline 11 & 85 & M & $\mathrm{AD}$ & 98 & 6 & 11 & 23 & 6 & 11 & 30 & 0 & 0 & 7 \\
\hline 12 & 89 & F & $\mathrm{AD}+\mathrm{PD}$ & 28 & 3 & 14 & 5 & 3 & 17 & 4 & 0 & 3 & -1 \\
\hline 13 & 89 & F & $\mathrm{AD}$ & 98 & 3 & 14 & 14 & 3 & 11 & 17 & 0 & -3 & 3 \\
\hline 14 & 90 & F & $\mathrm{AD}$ & 70 & 4 & 14 & 3 & 4 & 16 & 1 & 0 & 2 & -2 \\
\hline 15 & 90 & M & Not specified & 70 & 6 & 9 & 21 & 6 & 8 & 17 & 0 & -1 & -4 \\
\hline 16 & 91 & M & $\mathrm{AD}$ & 90 & 2 & 21 & 7 & 2 & 29 & 6 & 0 & 8 & -1 \\
\hline 17 & 91 & M & $\mathrm{AD}$ & 39 & 2 & 22 & 8 & 2 & 27 & 5 & 0 & 5 & -3 \\
\hline 18 & 94 & F & $\mathrm{AD}$ & 36 & 6 & 4 & 16 & 6 & 10 & 12 & 0 & 6 & -4 \\
\hline
\end{tabular}

Table 1. Patients' profiles. VD, Vascular dementia; AD, Dementia due to Alzheimer's disease; PD, Parkinson's diseases.

\section{Results}

Behavioural assessments. The average MMSE-J score was $12.4 \pm 6.6$ at the beginning and $14.4 \pm 7.8$ at the end of the NPT period (Table 1). The score increased (i.e. the cognitive level improved) in 10 patients, decreased in 5 patients, and remained unchanged in 3 patients. The mean score was significantly increased at the group level ( $p=0.002$, using bootstrap method). The changes in the MMSE-J score did not correlate with the patients' age, duration of the NPT period, or the initial MMSE-J score. Taken together, the cognitive functions of the study population improved over the NPT period but the patients' age nor their initial cognitive function was a predictor of this change.

The average DBD-13 score changed from $10.6 \pm 10.8$ at the beginning of the NPT period to $9.9 \pm 11.1$ during its end. This score decreased (i.e. behavioural problems were alleviated) in 12 patients, increased in 4 patients, and remained unchanged in 2 patients. The improvement of the DBD-13 score was not statistically significant ( $p=0.132$, using bootstrap method) at the group level. The change in the DBD-13 score did not correlate with age, duration of the NPT, or initial DBD-13 score.

The MMSE-J and DBD-13 scores were negatively correlated at the beginning $(p=0.010$, using bootstrap method) and the end ( $p=0.004$, using bootstrap method) of the NPT period. However, the changes in MMSE-J and DBD-13 scores showed no statistically significant correlation ( $p=0.084$, using bootstrap method).

Changes in spontaneous neural oscillations. The source intensities were compared between the two scans separately at each frequency band. The alpha1 and alpha2 intensities decreased at the second scan in the right temporal lobe (alpha1: $p=0.048$, alpha2: $p=0.049$ at the cluster-level), whereas only the alpha2 intensity exhibited a decline in the right fusiform gyrus $(p=0.028$ at the cluster-level) (Fig. 1 and Table 2). Moreover, following the NPT period, the low-gamma intensity in the right angular gyrus was increased ( $p=0.044$ at the cluster-level). The results in all other frequency bands did not reveal any significant changes.

Correlations of the MEG source intensities to the interval between the two scans and the changes in behavioural scores (MMSE-J and DBD-13) were also examined. Changes in beta source intensity close to the sensorimotor area of the right hemisphere were positively correlated with changes in DBD-13 scores $(p=0.028$ at the cluster-level). No other significant correlations were found between source intensities and the time between the scans or the behavioural scores. These results indicate that patients with reduced beta intensity near the right sensorimotor area showed a decline in behavioural disturbances.

To determine whether the NPT induced different effects in patients with vascular dementia (VD) and Alzheimer's disease (AD), we averaged the source intensity changes for patients with VD and AD separately and compared them visually (Fig. 2). Although there were minor differences in changes between VD and AD, they 


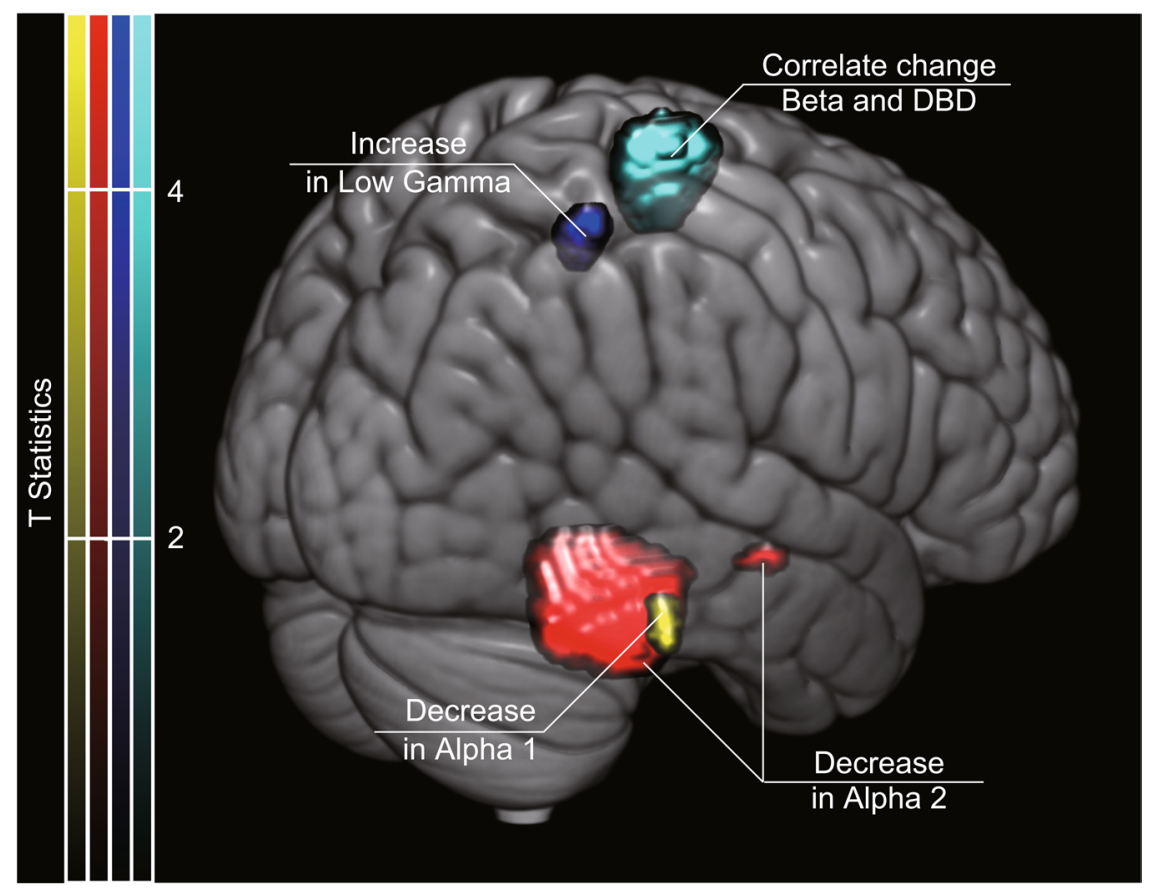

Figure 1. Brain regions with changes in source intensity after the NPT period. Red and yellow areas represent regions in which source intensities decreased after the NPT period. The blue area represents the region with an increase in source intensity after the NPT period. The area in cyan represents the region in which the change in source intensity was positively correlated with the change in the DBD-13 score. The 3D image was created using MRIcroGL (https://www.mccauslandcenter.sc.edu/mricrogl/).

\begin{tabular}{|c|c|c|c|c|c|c|c|c|c|}
\hline \multirow[b]{2}{*}{ Change } & \multirow[b]{2}{*}{ Frequency } & \multicolumn{2}{|c|}{ Cluster level } & \multicolumn{2}{|c|}{ Peak level } & \multicolumn{3}{|c|}{ Coordinate } & \multirow[b]{2}{*}{ Brain region } \\
\hline & & $\mathbf{P}(\mathrm{FWE})$ & $\mathbf{k E}$ & P(FWE) & $\mathbf{T}$ & $\mathbf{x}$ & $\mathbf{Y}$ & $\mathbf{Z}$ & \\
\hline \multirow{3}{*}{ Decrease after NPT } & Alpha1 & 0.048 & 25 & 0.047 & 4.63 & 52 & -38 & -20 & Right Inferior Temporal Gyrus \\
\hline & Alpha2 & 0.028 & 858 & 0.014 & 5.58 & 40 & -46 & -16 & Right Fusiform Gyrus \\
\hline & & 0.049 & 14 & 0.047 & 4.78 & 48 & -14 & -12 & Right Superior Temporal Gyrus \\
\hline Increase after NPT & Low Gamma & 0.044 & 72 & 0.032 & 5.13 & 42 & -52 & 60 & Right Angular Gyrus \\
\hline Positive correlate with DBD-13 & Beta & 0.028 & 504 & 0.016 & 5.63 & 30 & -28 & 70 & Right Sensorimotor Area \\
\hline
\end{tabular}

Table 2. Intensity changes the resting-state brain activity between the two scans (Corresponding to Fig. 1). The $\mathrm{p}$-values were corrected for multiple comparisons by the Family-wise-error (FWE) correction. T, $t$-value; $p$, p-value; X, X-coordinate; Y, Y-coordinate; Z, Z-coordinate.

mostly overlapped and shared the same regions as those shown in Fig. 1. Patients with AD showed significant change in source intensity (Table 3); conversely, the changes in the VD were not significant.

\section{Discussion}

The present study revealed two major findings: (1) NPTs improved cognitive functions in patients with dementia, although behavioural disturbance parameters were not significantly modified, and (2) the spontaneous neural oscillations (i.e. the resting-state brain activity) in the right hemisphere changed over the NPT period and parts of these changes correlated with changes in the behavioural score (DBD-13).

It has been suggested that brains of patients with dementia are severely damaged thus negatively influencing brain plasticity. This is considered to be the reason why these patients do not respond to pharmacological treatments ${ }^{5}$. This idea is supported by the fact that patients with CIND and MCI show improvements in their cognitive functions after NPTs ${ }^{18-23}$, and neuroimaging studies have revealed that these patients still show signs of brain plasticity ${ }^{16,17}$. The present study applied this idea to patients with dementia. We demonstrated that patients with dementia responded to NPT and that the intervention improved the cognitive functions, as reflected by the MMSE-J score. This improvement did not correlate with either age nor initial MMSE-J score, indicating that the potential of NPT is not limited to young patients or patients with mild dementia. A previous study demonstrated that pathological changes are not always in agreement with the clinical symptoms ${ }^{24}$; patients with less brain damage may exhibit more severe dementia symptoms and vice versa. It is, however, reasonable to assume that some patients with dementia have the potential to improve their functions similar to that of patients with CIND or MCI. 


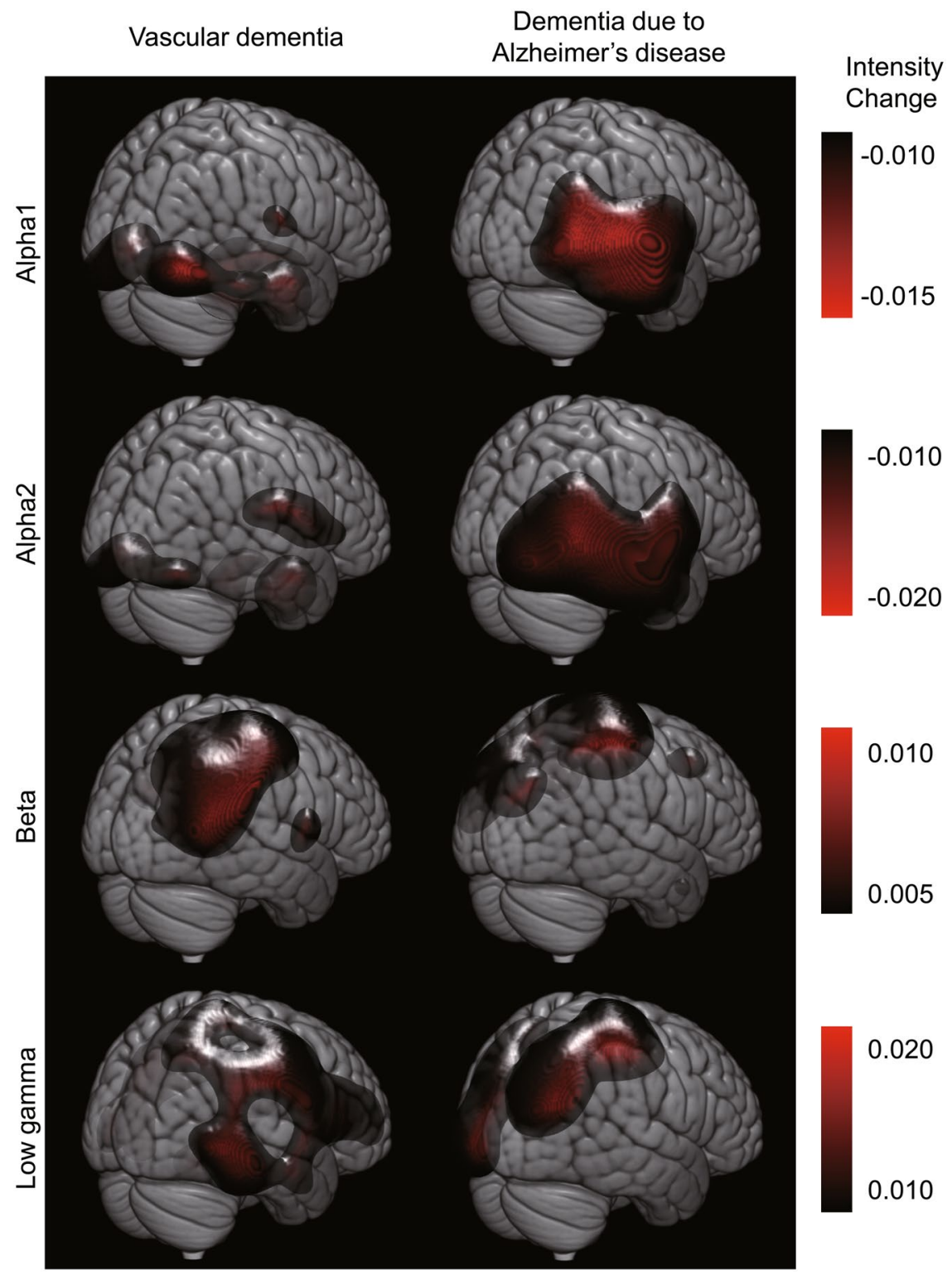

Figure 2. Averaged changes in source intensity for VD and AD (corresponding to Fig. 1). Upper and lower limits of the colour bars correspond to the display threshold. The 3D image was created using MRIcroGL (https://www.mccauslandcenter.sc.edu/mricrogl/).

\begin{tabular}{|c|c|c|c|c|c|c|c|c|c|}
\hline \multirow[b]{2}{*}{ Change } & \multirow[b]{2}{*}{ Frequency } & \multicolumn{2}{|c|}{ Cluster level } & \multicolumn{2}{|c|}{ Peak level } & \multicolumn{3}{|c|}{ Coordinate } & \multirow[b]{2}{*}{ Brain region } \\
\hline & & P (FWE) & kE & $P$ (unc) & $T$ & $\mathrm{X}$ & $\mathbf{Y}$ & $\mathbf{Z}$ & \\
\hline \multirow{2}{*}{$\begin{array}{l}\text { Decrease } \\
\text { after NPT }\end{array}$} & Alphal & 0.201 & 256 & 0.001 & 4.47 & 56 & -38 & -24 & \begin{tabular}{|l} 
Right Inferior \\
Temporal \\
Gyrus
\end{tabular} \\
\hline & Alpha2 & 0.083 & 4942 & $>0.001$ & 4.28 & 50 & -22 & -10 & $\begin{array}{l}\text { Right Superior } \\
\text { Temporal } \\
\text { Gyrus }\end{array}$ \\
\hline $\begin{array}{l}\text { Increase } \\
\text { after NPT }\end{array}$ & Low Gamma & 0.242 & 477 & $>0.001$ & 3.81 & 38 & -56 & 60 & $\begin{array}{l}\text { Right Angular } \\
\text { Gyrus }\end{array}$ \\
\hline \begin{tabular}{|l} 
Positive \\
correlate \\
with DBD- \\
13
\end{tabular} & Beta & 0.287 & 388 & $>0.001$ & 4.79 & 26 & -24 & 72 & $\begin{array}{l}\text { Right } \\
\text { Sensorimotor } \\
\text { Area }\end{array}$ \\
\hline
\end{tabular}

Table 3. Intensity changes for patients with dementia due to Alzheimer's diseases (corresponding to Table 2). The frequency bands and corresponding brain regions, where the differences reached a statistically significant level, are displayed. unc, uncorrected; T, t-value; $\mathrm{p}$, p-value; $\mathrm{kE}$, cluster size; X, X-coordinate; Y, Y-coordinate; Z, Z-coordinate. 
Previous studies have shown that NPTs have beneficial effects on healthy older adults ${ }^{10,25}$. The effects of NPTs were independent of the pathological changes in the brain during disease state causing dementia, therefore NPTs are effective in patients with different subtypes of dementia such as VD and dementia due to AD. This is consistent with the novel concept of NPT, which focuses on each patient's function rather than pathophysiological or neurological causes of their disease ${ }^{21}$. In the present study, we further demonstrated that NPT induced similar changes in the brain activities of patients with either VD or dementia due to AD (see Fig. 2 and Table 3). The primary results (Fig. 1 and Table 2) show the common changes that underlie different pathologies relevant to dementia. NPTs induce neuroplasticity or change the neural network efficiency to compensate for brain functional deficit during disease state ${ }^{25,26}$. A previous study using MRI in healthy older adults showed that NPT changes grey matter densities in the postcentral gyrus, right hippocampus, and superior temporal gyrus ${ }^{27}$. The grey matter density in the postcentral gyrus is modulated by NPT ${ }^{27}$ and the extent of these changes correlate with the NPT outcome. The postcentral gyrus is a part of the sensorimotor area where neural activities are affected by ageing and dementia, and where changes are modulated by and associated with subcortical changes ${ }^{28}$. In the present study, changes in beta intensity in the sensorimotor area correlated with the observed changes in the scores measuring behavioural disturbances. Beta intensity is associated with gamma-aminobutyric acid (GABAergic) neuronal activities ${ }^{29}$ which is correlated to PPSD $^{30}$ and ultimately, neural plasticity ${ }^{31}$. It is plausible that changes in beta intensity in the sensorimotor area reflect the changes in neuroplasticity or neural network efficiency caused by NPTs through the whole brain network system. Another study using functional MRI in healthy older adults revealed that NPT changes brain activities in a widespread network of frontal, parietal, temporal, subcortical, and occipital regions as well as in the thalamus and the caudate ${ }^{10}$. This is supported by additional studies using functional MRI in patients with MCI showing that NPTs modify brain activities within a large network that includes the frontal, temporal, and parietal areas ${ }^{12}$. In the present study, the resting-state activity of the alpha band was altered in the right temporal lobe and the right fusiform gyrus. A larger part of the alpha activity is produced by reciprocal connections between the neocortex and the thalamus ${ }^{32,33}$. Previous studies indicate that NPTs modulate large neural networks including the thalamus ${ }^{10,12}$. The temporal lobe and the fusiform gyrus are anatomically close to the hippocampus and the right hippocampus has been implicated in dementia-related psychosis ${ }^{34}$. NPT effectively reduces BPSD ${ }^{35}$, thus it is a reasonable expectation that NPT alters the spontaneous neural oscillation of the alpha band in the right temporal lobe and the fusiform gyrus. A functional MRI study has shown that NPTs induced brain activity changes in the right angular gyrus and the anterior portion of the left lingual gyrus of patients with $\mathrm{AD}^{36}$. Our result showed that gamma intensity in the right angular gyrus increased after NPT. The angular gyrus is one of the brain network cortical hubs ${ }^{37}$ which influences various cognitive functions. It is located in the parietal cortex where ageing and dementia bring about changes at an early stage (Retorogenesis theory $)^{38,39}$. Lesions in the angular gyrus cause symptoms similar to those in BPSD ${ }^{40,41}$. Gamma activity is also correlated with the activities of GABAergic neurons ${ }^{42}$, and a reduction in GABA is associated with poor cognition $^{43,44}$. We speculate that changes in gamma at the angler gyrus reflected the change in neural network efficiency induced by NPTs to compensate for the functional deficit.

In the present study, we used MEG data instead of MRI or functional MRI data. MEG is a non-invasive neuroimaging technique that is sensitive to changes in brain activity related to dementia ${ }^{45-47}$ as it can detect synaptic transmission underlying macroscopically observable brain activity ${ }^{48}$. In dementia research, MEG demonstrated various advantages over MRI. In practice, MRI scans are often difficult and risky for patients with dementia ${ }^{36}$. For example, patients with dementia unintentionally bring metallic items into the scanner at times, which is highly dangerous due to the strong magnetic field produced by an MRI scanner. It takes up to $10 \mathrm{~s}$ to abort a scan and rescue the patient in the case of an emergency. Moreover, patients are often not happy with an MRI scan as the device creates loud noises. The patients also feel nervous in the confined space of the MRI gantry and must keep still inside the scanner without having any company. By contrast, a MEG generates neither a magnetic field nor noise. Patients are not opposed to this technique as they are not physically restricted by the scanner and another person can attend to them during the scan to ensure their safety. A scan is completed within 10 min or less including preparations, can be immediately aborted anytime, and in case of an emergency, the patient can be taken out within $10 \mathrm{~s}$. Additionally, MEG scans provide clinicians with an opportunity to detect epileptic discharges. Of patients with dementia, 10-22\% have epilepsy as a secondary diagnosis and it is not easy to diagnose epilepsy in older adults because their seizures are often non-convulsive ${ }^{49-52}$. In fact, in the present study, 2 out of the 21 patients presented epileptic discharges during the scan and were excluded from further analyses so that they may be treated with anti-epileptic drugs. In summary, the present study restated that MEG is an ideal neuroimaging technique for dementia studies.

This study has five limitations: (1) The group of patients with dementia was heterogeneous in terms of their pathologies of the brain diseases causing dementia (e.g. stroke or Alzheimer's diseases), thus making it challenging to fully evaluate how NPTs affected pathological changes of the diseased brain. However, as shown in Fig. 2 and Table 3, NPTs effects are independent of pathological changes and thus highlight the NPT's common effect on dementia in general, which is a heterogeneous syndrome. (2) We did not address the effects of inter-individual factors such as patient's age, sex, genotypes, brain diseases causing dementia, and batteries of NPTs. This study aimed to determine whether NPTs changes brain activities (not pathology) and focused on the intra-individual relationship between cognitive/behavioural scores and spontaneous neural oscillations. Contributions of inter-individual factors should be addressed in future studies. (3) We did not include control participants since this was an observational study at a geriatric health services facility. It might be possible that the changes in resting-state activities were caused by spontaneous recovery rather than by NPT. However, the intensity changes in beta-band activity over the sensorimotor area correlated with the changes in DBD-13 scores and the duration of the NPT did not have any significant effects on resting-state brain activities. Therefore, it seems plausible that the changes in the resting-state brain activities were caused by the NPT. (4) The sample size is limited. We avoided generalising our results to NPTs in patients with all kinds of dementia. What we have demonstrated here is that 


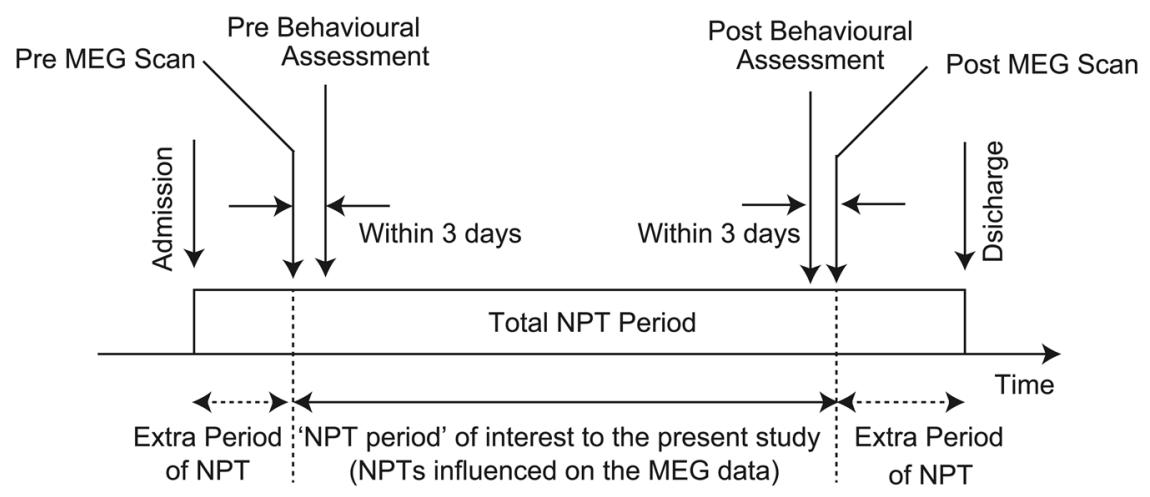

Figure 3. Time course of the present study. MEG scans and behavioural assessments and were carried out two times during non-pharmacological treatment (NPT) period. Each MEG scan and its corresponding behavioural assessments were obtained within 3 days. The interval between the two MEG scans was defined as the NPT period. Some patients were involved in 'extra' NPT programmes (before their first MEG scan and/or after the second MEG scan), it is not relevant to the present study.

some patients with dementia have the potential to improve their brain functions and resting-state brain activities following NPTs and is largely consistent with previous studies investigating brain plasticity in older adults and patients at risk of dementia. (5) The change in oscillation intensity did not correlate with the change in cognitive scores (MMSE-J). MMSE-J consists of 11 major items targeting different cognitive functions (temporal orientation, spatial orientation, immediate memory, attention/concentration, delayed recall, naming, verbal repetition, verbal comprehension, writing, reading a sentence, and constructional praxis). Each patient changed their scores in different items. In addition, the change in the total score of MMSE-J may not reflect cognitive improvement linearly. The change from 25 to 26 could be bigger than that from 15 to 16 as a change in cognitive function. These two changes could be based on neurological changes in different cortical areas. We can assume that the heterogonous improvement and non-linearity prevented us from observing a statistically significant correlation between changes in the oscillation intensity and the MMSE-J sore.

Taken together, NPTs can change the resting-state brain activity and improve dementia symptoms. These changes are based on neural plasticity and not limited to patients in pre-dementia conditions (CIND and MCI). Clinicians and therapists should pay more attention to primary care patients with dementia because these patients have the potential for improvement with NPTs.

\section{Methods}

Patients, rehabilitation procedure, and ethics. A total of 21 patients with dementia (12 females, 9 males; age, average \pm standard deviation [SD]: $85.4 \pm 5.4$ years) were enrolled from our geriatric health services facility 'Kakehashi' regardless of the brain disease causing dementia (e.g. stroke or AD). The group of patients with dementia was an epitome of the population of dementia in the region. The dementia diagnosis was established by clinicians prior to patients' admission to the facility. Most patients were affected by AD with other types of dementia also being present in the study population (see Table 1). Medication schedules established prior to admission of the patient to our facility were continued unchanged throughout the NPT period in the facility, if possible. Usually, patients stayed in the facility for several months (average \pm SD: $83.9 \pm 32.7$ days, ranging from 30 to 147 days) where they were treated every day by the NPT therapists. NPT consisted of five major activities: (1) physical exercise (e.g. regular daily activity training or ergometer cycling), (2) therapeutic role-playing (e.g. reminiscence therapy), (3) nursing care (e.g. proper eating, drinking and keeping sanitary environment), (4) horticultural therapy, and (5) self-cognitive training (e.g. colouring books or crossword puzzles). All patients participated in the activities (1) - (3), whereas (4) and (5) were optional activities. The battery of these NPTs and the details of the activities were individually adjusted daily by the NPT therapists after considering patients' physical, mental, and cognitive conditions. The length of the daily NPT programmes [i.e. (1), (2) and (4)] was in accordance with the Japanese regulations: 20-40 min, depending on the patients' condition. During the patients' stays in the facility, dietary and hygienic regimens were provided by nurses, dental hygienists, and nutritionists. All patients with their families gave written informed consent to participate in this study. This study was conducted in accordance with the Declaration of Helsinki and was approved by the Ethics Committee of Hokuto Hospital.

Behavioural assessments. The levels of cognitive impairments and behavioural disturbances in patients were assessed by therapists using the Japanese version of the Mini-Mental State Examination (MMSE-J) $)^{53,54}$ and a short version of the Dementia Behaviour Disturbance Scale (DBD-13) $)^{55}$. Each patient completed a set of behavioural assessments (MMSE-J and DBD-13, conducted on the same day) two times during NPT period (Fig. 3). The interval between the two tests was several months (average \pm SD: $74.7 \pm 32.1$ days, ranging from 26 to 132 days). The MMSE-J is a 30-point cognitive test. In this test, a lower score indicates a more severe cognitive impairment ${ }^{56}$, and dementia is suspected when the score is below 23. The DBD-13 is a 52-point behavioural rating scale and consists of 13 items (item \#1,2,3,4,5,15,16,17,21, 24, 26, 27, and 28 of the original version of the Dementia Behaviour Disturbance Scale ${ }^{57}$ ) and a lower score indicates a less problematic behaviour (such as agitation, aggression, anxiety, or apathy). There is no reference value for the DBD-13. 
MEG scanning. All patients visited our MEG centre at Hokuto Hospital two times during the NPT period (Fig. 3). The 3-minute travel was comfortable by car between the geriatric health services facility and the MEG centre. The interval between the two scans was a few months (average \pm SD: $75.0 \pm 29.7$ days, ranging from 28 to 133 days). Each MEG scan and its corresponding behavioural assessments were obtained within 3 days. The two intervals between the two behaviour assessments and two MEG scans were almost identical. The interval between the two MEG scans was defined as the NPT period. In the present study, some patients were involved in the NPT programmes before the first MEG scan, while others were involved after the second MEG scan; however, these "extra" periods were not considered in our study (Fig. 3). Spontaneous neural oscillations (i.e. resting-state brain activity) were recorded for $5 \mathrm{~min}$ using a 160-channel whole-head type magnetoencephalography system (MEG vision PQ1160C; Yokogawa, Kanazawa, Japan). During the scan, patients were asked to calmly stay in the supine position with eyes closed in a magnetically shielded room. The scanning condition was controlled to be as consistent and comfortable as possible. For safety reasons, another person remained next to the patient. The sensor and reference coils were gradiometers of $15.5 \mathrm{~mm}$ in diameter and $50 \mathrm{~mm}$ in the baseline, and each pair of sensor coils was separated by a distance of $23 \mathrm{~mm}$. The sampling frequency was $1,000 \mathrm{~Hz}$ with $200 \mathrm{~Hz}$ low-pass filtering during the recording. To co-register MEG source images with structural brain images acquired by standard magnetic resonance imaging (MRI), three fiducial magnetic marker coils were placed on the patients' face ( $5 \mathrm{~mm}$ above the nasion and bilaterally $10 \mathrm{~mm}$ in front of the tragus) during the MEG scan.

MEG analysis. Of the 21 patients, 3 patients were excluded from analysis: 2 exhibited epileptic discharges during the MEG scans and 1 induced severe artefacts due to dyskinesia. The remaining 18 patients were analysed offline using the software package SPM-12 (Wellcome Trust Centre for Neuroimaging, London, UK; https:// www.fil.ion.ucl.ac.uk/spm/) and the MEAW system (https://www.hokuto7.or.jp/hospital/lang/english-home/ meaw/) (Table 1). For the ease of analysis, the continuous MEG signals were divided into $10 \mathrm{~s}$ segments. Epochs in which the magnetic signal exceeded 6,000 fT, were discarded. Since the experimental environment generated a utility frequency, a $50-\mathrm{Hz}$ band-stop filter was applied to the epoched data. These filtered data were directly used for source-level analyses. To identify the locations of the brain producing the resting-state-induced component, the source inversion procedures were applied to the oscillation components of delta $(0-3 \mathrm{~Hz})$, theta $(4-7 \mathrm{~Hz})$, alpha1 $(7-9 \mathrm{~Hz})$, alpha2 $(9-11 \mathrm{~Hz})$, alpha3 $(11-13 \mathrm{~Hz})$, beta $(13-25 \mathrm{~Hz})$, and gamma (low gamma, $26-40 \mathrm{~Hz}$; high gamma, $41-80 \mathrm{~Hz}$ ) separately, using a maximal smoothness algorithm with a spatially coherent sources model (i.e. $\mathrm{COH}$ algorithms implemented in SPM-12) ${ }^{58}$, which is comparable to sLORETA ${ }^{59}$. The COH algorithm is a popular source inversion algorithm and is often used in clinical environments ${ }^{60,61}$. Forward modelling was performed for the whole brain using a single shell model with canonical MR images provided by SPM-12. The source inversion and estimation were performed by applying filters which corresponded to each frequency band (from delta to high gamma). No source priors were used for source estimation. The source images were smoothed $(20 \times$ $20 \times 20 \mathrm{~mm}$ ) and taken to the second (group)-level analysis.

Two types of the second (group)-level analyses were carried out: (1) to find brain regions in which intensities of the spontaneous neural oscillation were different between the two scans, and (2) to find brain regions in which changes in source intensities were correlated with changes in MMSE-J scores, DBD-13 scores, or the time between the two scans. For the first analysis, the source images were compared within each patient using the paired $t$-test at each frequency band with the three covariates MMSE-J scores, DBD-13 scores, and the time between the two scans. Both positive and negative effects of these covariates on the source intensities were evaluated by building $t$-contrasts with +1 and -1 . For the second analysis, source images were created to represent changes in source intensities between the two scans using Image Calculator, which is an SPM-12 tool. These images were analysed using the one-sample $t$-test with a covariate of the change in the scores of MMSE-J or DBD-13 or the time between the two scans. Here, we report the source locations of peak level activations at a significance threshold of $p($ corrected for family-wise error $[F W E])=0.05$ and a cluster extent at $\mathrm{k}>10\left(=80 \mathrm{~mm}^{3}\right)^{62}$. Cortical areas at which the peaks of the estimated sources were located were identified using the SPM-12 software.

After the main analyses, we performed two additional analyses to determine whether the NPT induced different effects on patients with VD $(\mathrm{n}=4)$ and $\mathrm{AD}(\mathrm{n}=11)$. For the first step, the changes in source intensities between the two scans were averaged over patients with the same pathological labelling (VD and AD, separately) using the Image Calculator; the averaged images were visually inspected (Fig. 2). For the second step, for both the $\mathrm{VD}$ and $\mathrm{AD}$ patient groups, the source intensities were compared within each patient using the paired t-test for each frequency band (the procedures are same as the main analysis described above). Here, we report the source locations of peak level activations at a significance threshold of $p$ (uncorrected) $=0.001$ and a cluster extent at $\mathrm{k}>10$.

Statistical analysis. Bootstrapping approaches were used to evaluate changes in MMSE-J and DBD-13 scores over NPT period considering a relatively small number of patients $(n=18)$. First, changes in these scores between pre- and post-NPT were evaluated. The difference between the pre- and post-NPT scores was resampled with 10,000 times replacement across the 18 patients, and the percentage of the resampled differences, being larger or smaller than 0 (the smaller value), was taken as the significance level. Second, the correlations between the patients' age, the duration of the NPT period, and the MMSE-J and DBD-13 scores were evaluated. For each pair of variables, the correlation coefficient was calculated from resampled with replacement data across the 18 patients for 10,000 times, and percentage of the resampled coefficients, being larger or smaller than 0 (the smaller value), was taken as the significance level. Statistical analyses were performed using MATLAB (MathWorks, MA, USA). 


\section{Data availability}

The datasets generated during and/or during the current study are available from the corresponding author on reasonable request.

Received: 3 July 2019; Accepted: 7 April 2020;

Published online: 21 April 2020

\section{References}

1. Shaji, K. S., Sivakumar, P. T., Rao, G. P. \& Paul, N. Clinical Practice Guidelines for Management of Dementia. Indian J. Psychiatry 60, S312-S328 (2018).

2. Qaseem, A. et al. Current pharmacologic treatment of dementia: a clinical practice guideline from the American College of Physicians and the American Academy of Family Physicians. Ann. Intern. Med. 148, 370-8 (2008).

3. Zucchella, C. et al. The Multidisciplinary Approach to Alzheimer's Disease and Dementia. A Narrative Review of NonPharmacological Treatment. Front. Neurol. 9, 1058 (2018).

4. Egan, M. F. et al. Randomized Trial of Verubecestat for Prodromal Alzheimer's Disease. N. Engl. J. Med. 380, 1408-1420 (2019).

5. Lo, R. Y. The borderland between normal aging and dementia. Ci ji yi xue za zhi 29, 65-71 (2017).

6. Robinson, L., Tang, E. \& Taylor, J.-P. Dementia: timely diagnosis and early intervention. BMJ 350, h3029-h3029 (2015).

7. Leifer, B. P. Early Diagnosis of Alzheimer's Disease: Clinical and Economic Benefits. J. Am. Geriatr. Soc. 51, S281-S288 (2003).

8. Almkvist, O. \& Winblad, B. Early diagnosis of Alzheimer dementia based on clinical and biological factors. Eur. Arch. Psychiatry Clin. Neurosci. 249(Suppl), 3-9 (1999).

9. Freter, S., Bergman, H., Gold, S., Chertkow, H. \& Clarfield, A. M. Prevalence of potentially reversible dementias and actual reversibility in a memory clinic cohort. CMAJ 159, 657-62 (1998).

10. Belleville, S. et al. Improvement of Episodic Memory in Persons with Mild Cognitive Impairment and Healthy Older Adults: Evidence from a Cognitive Intervention Program. Dement. Geriatr. Cogn. Disord. 22, 486-499 (2006).

11. Belleville, S. Cognitive training for persons with mild cognitive impairment. Int. psychogeriatrics 20, 57-66 (2008).

12. Belleville, S. et al. Training-related brain plasticity in subjects at risk of developing Alzheimer's disease. Brain 134, 1623-1634 (2011).

13. Blumenthal, J. A. et al. Lifestyle and neurocognition in older adults with cognitive impairments: A randomized trial. Neurology 92 , e212-e223 (2019).

14. Petersen, R. C. et al. Mild cognitive impairment: a concept in evolution. J. Intern. Med. 275, 214-28 (2014).

15. de Jager, C. A. \& Budge, M. M. Stability and predictability of the classification of mild cognitive impairment as assessed by episodic memory test performance over time. Neurocase 11, 72-9 (2005).

16. Nagamatsu, L. S., Handy, T. C., Hsu, C. L., Voss, M. \& Liu-Ambrose, T. Resistance training promotes cognitive and functional brain plasticity in seniors with probable mild cognitive impairment. Arch. Intern. Med. 172, 666-8 (2012).

17. Cabeza, R. Hemispheric asymmetry reduction in older adults: the HAROLD model. Psychol. Aging 17, 85-100 (2002).

18. Spector, A. et al. Efficacy of an evidence-based cognitive stimulation therapy programme for people with dementia: randomised controlled trial. Br. J. Psychiatry 183, 248-54 (2003).

19. Huntley, J. D., Gould, R. L., Liu, K., Smith, M. \& Howard, R. J. Do cognitive interventions improve general cognition in dementia? A meta-analysis and meta-regression. BMJ Open 5, e005247 (2015).

20. Toba, K. et al. Intensive rehabilitation for dementia improved cognitive function and reduced behavioral disturbance in geriatric health service facilities in Japan. Geriatr. Gerontol. Int. 14, 206-11 (2014).

21. Maki, Y., Sakurai, T., Okochi, J., Yamaguchi, H. \& Toba, K. Rehabilitation to live better with dementia. Geriatr. Gerontol. Int. 18, 1529-1536 (2018).

22. Douglas, S., James, I. \& Ballard, C. Non-pharmacological interventions in dementia. Adv. Psychiatr. Treat. 10, 171-177 (2004).

23. Lord, T. R. \& Garner, J. E. Effects of Music on Alzheimer Patients. Percept. Mot. Skills 76, 451-455 (1993).

24. Ince, P. Pathological correlates of late-onset dementia in a multicentre, community-based population in England and Wales. Neuropathology Group of the Medical Research Council Cognitive Function and Ageing Study (MRC CFAS). Lancet (London, England) 357, 169-75 (2001).

25. Lampit, A., Hallock, H., Suo, C., Naismith, S. L. \& Valenzuela, M. Cognitive training-induced short-term functional and long-term structural plastic change is related to gains in global cognition in healthy older adults: a pilot study. Front. Aging Neurosci. 7 (2015).

26. Stern, Y. Cognitive reserve. Neuropsychologia 47, 2015-28 (2009).

27. Lampit, A., Hallock, H., Suo, C., Naismith, S. L. \& Valenzuela, M. Cognitive training-induced short-term functional and long-term structural plastic change is related to gains in global cognition in healthy older adults: a pilot study. Front. Aging Neurosci. 7, 14 (2015).

28. Cheng, C.-H., Chan, P.-Y. S., Baillet, S. \& Lin, Y.-Y. Age-Related Reduced Somatosensory Gating Is Associated with Altered Alpha Frequency Desynchronization. Neural Plast. 2015, 302878 (2015).

29. JENSEN, O. et al. On the human sensorimotor-cortex beta rhythm: Sources and modeling. Neuroimage 26, 347-355 (2005).

30. Lanctôt, K. L., Herrmann, N., Mazzotta, P., Khan, L. R. \& Ingber, N. GABAergic function in Alzheimer's disease: evidence for dysfunction and potential as a therapeutic target for the treatment of behavioural and psychological symptoms of dementia. Can. J. Psychiatry. 49, 439-53 (2004).

31. Griffen, T. C. \& Maffei, A. GABAergic synapses: their plasticity and role in sensory cortex. Front. Cell. Neurosci. 8, 91 (2014).

32. Steriade, M. Corticothalamic resonance, states of vigilance and mentation. Neuroscience 101, 243-276 (2000).

33. Vecchio, F. et al. Electroencephalographic rhythms in alzheimer's disease. Int. J. Alzheimers. Dis. 2011, (2011).

34. Lee, K. et al. Right hippocampus atrophy is independently associated with Alzheimer's disease with psychosis. Psychogeriatrics 19, 105-110 (2019).

35. Dyer, S. M., Harrison, S. L., Laver, K., Whitehead, C. \& Crotty, M. An overview of systematic reviews of pharmacological and nonpharmacological interventions for the treatment of behavioral and psychological symptoms of dementia. Int. Psychogeriatrics 30, 295-309 (2018).

36. Satoh, M. et al. Music Therapy Using Singing Training Improves Psychomotor Speed in Patients with Alzheimer's Disease: A Neuropsychological and fMRI Study. Dement. Geriatr. Cogn. Dis. Extra 5, 296-308 (2015).

37. Seghier, M. L. The angular gyrus: multiple functions and multiple subdivisions. Neuroscientist 19, 43-61 (2013).

38. Reisberg, B. et al. Retrogenesis: Clinical, physiologic, and pathologic mechanisms in brain aging, Alzheimer's and other dementing processes. Eur. Arch. Psychiatry Clin. Neurosci. 249, 28-36 (1999).

39. Reisberg, B. et al. Evidence and mechanisms of retrogenesis in Alzheimer's and other dementias: Management and treatment import. Am. J. Alzheimers. Dis. Other Demen. 17, 202-211 (2002).

40. Benson, D. F. \& Cummings, J. L. Angular gyrus syndrome simulating Alzheimer's disease. Arch. Neurol. 39, 616-20 (1982).

41. Nagaratnam, N., Phan, T. A., Barnett, C. \& Ibrahim, N. Angular gyrus syndrome mimicking depressive pseudodementia. J. Psychiatry Neurosci. 27, 364-8 (2002).

42. Bartos, M., Vida, I. \& Jonas, P. Synaptic mechanisms of synchronized gamma oscillations in inhibitory interneuron networks. Nat. Rev. Neurosci. 8, 45-56 (2007). 
43. Porges, E. C. et al. Frontal Gamma-Aminobutyric Acid Concentrations Are Associated With Cognitive Performance in Older Adults. Biol. psychiatry. Cogn. Neurosci. neuroimaging 2, 38-44 (2017).

44. Mably, A. J. \& Colgin, L. L. Gamma oscillations in cognitive disorders. Curr. Opin. Neurobiol. 52, 182-187 (2018).

45. Fernández, A. et al. Focal temporoparietal slow activity in Alzheimer's disease revealed by magnetoencephalography. Biol. Psychiatry 52, 764-70 (2002).

46. Criado, J. R., Amo, C., Quint, P., Kurelowech, L. \& Otis, S. M. Using magnetoencephalography to study patterns of brain magnetic activity in Alzheimer's disease. Am. J. Alzheimers. Dis. Other Demen. 21, 416-23 (2007).

47. Gómez, C., Abásolo, D., Poza, J., Fernández, A. \& Hornero, R. MEG analysis in Alzheimer’s disease computing approximate entropy for different frequency bands. In 2010 Annual International Conference of the IEEE Engineering in Medicine and Biology 2010, 2379-2382 (IEEE, 2010).

48. van Straaten, E. C. W. et al. Magnetoencephalography for the detection of intervention effects of a specific nutrient combination in patients with mild Alzheimer's disease: Results from an exploratory double-blind, randomized, controlled study. Front. Neurol. 7 (2016).

49. Colom, L. V. Septal networks: relevance to theta rhythm, epilepsy and Alzheimer's disease. J. Neurochem. 96, 609-623 (2006).

50. Chiara, C. et al. Nonconvulsive seizures and dementia: a case report. Int. J. Alzheimers. Dis. 2011, 690305 (2011).

51. Stephen, L. J. \& Brodie, M. J. Epilepsy in elderly people. Lancet 355, 1441-1446 (2000).

52. Scarmeas, N. et al. Seizures in Alzheimer Disease: Who, When, and How Common? Nikolaos. October 66, 992-997 (2009).

53. Folstein, M. F., Folstein, S. E. \& McHugh, P. R. Mini-mental state?. A practical method for grading the cognitive state of patients for the clinician. J. Psychiatr. Res. 12, 189-98 (1975).

54. Sugishita, M., Hemmi, I. \& Iwatsubo, T. Japanese versions equivalent to original English neuropsychological tests in ADNI. Alzheimer's Dement. 6, S348 (2010).

55. Machida, A. Estimation of the reliability and validity of the short version of the 28 -item Dementia Behavior Disturbance scale. Nihon Ronen Igakkai Zasshi 49, 463-467 (2012).

56. Pangman, V. C., Sloan, J. \& Guse, L. An examination of psychometric properties of the mini-mental state examination and the standardized mini-mental state examination: implications for clinical practice. Appl. Nurs. Res. 13, 209-13 (2000).

57. Baumgarten, M., Becker, R. \& Gauthier, S. Validity and reliability of the dementia behavior disturbance scale. J. Am. Geriatr. Soc. 38, $221-6(1990)$

58. Friston, K. et al. Multiple sparse priors for the M/EEG inverse problem. Neuroimage 39, 1104-20 (2008).

59. Pascual-Marqui, R. D. Standardized low-resolution brain electromagnetic tomography (sLORETA): technical details. Methods Find. Exp. Clin. Pharmacol. 24(Suppl D), 5-12 (2002).

60. Ray, A. \& Bowyer, S. Clinical applications of magnetoencephalography in epilepsy. Ann. Indian Acad. Neurol. 13, 14 (2010).

61. Terakawa, Y. et al. Quantitative analysis of MEG using modified sLORETA for clinical application. Clinical neurophysiology: official journal of the International Federation of Clinical Neurophysiology 119, 1917-22 (2008).

62. Lieberman, M. D. \& Cunningham, W. A. Type I and Type II error concerns in fMRI research: re-balancing the scale. Soc. Cogn. Affect. Neurosci. 4, 423-8 (2009).

\section{Acknowledgements}

This study is partially sponsored by RICOH. The sponsor has no role in study concept/design, methods, data analysis and preparation of paper. We sincerely thank all of the staff at Geriatric health services facility Kakehashi. We would like to thank Editage (www.editage.com) for English language editing.

\section{Author contributions}

Y.S. and H.K. planed the study and Y.S. designed the study protocol. K.S. managed patients and organised MEG scans. Y.S. and T.O. operated MEG system. Y.S. and H.H. analysed the data, wrote the main manuscript text and prepare the figure.

\section{Competing interests}

The authors declare no competing interests.

\section{Additional information}

Correspondence and requests for materials should be addressed to Y.S.

Reprints and permissions information is available at www.nature.com/reprints.

Publisher's note Springer Nature remains neutral with regard to jurisdictional claims in published maps and institutional affiliations.

Open Access This article is licensed under a Creative Commons Attribution 4.0 International License, which permits use, sharing, adaptation, distribution and reproduction in any medium or format, as long as you give appropriate credit to the original author(s) and the source, provide a link to the Creative Commons license, and indicate if changes were made. The images or other third party material in this article are included in the article's Creative Commons license, unless indicated otherwise in a credit line to the material. If material is not included in the article's Creative Commons license and your intended use is not permitted by statutory regulation or exceeds the permitted use, you will need to obtain permission directly from the copyright holder. To view a copy of this license, visit http://creativecommons.org/licenses/by/4.0/.

(c) The Author(s) 2020 